\title{
COUPLED NONLINEAR SYSTEM IN $(2+1)$ DIMENSION AND OVERTURNING SOLITONS
}

\author{
C. Ghosh ANd A.R. Chowdhury
}

High Energy Physics Division, Department of Physics Jadavpur University, Calcutta 700 032, India

(Received January 13, 1995; revised version June 19, 1995; in final form December 5, 1995)

\begin{abstract}
Coupled nonlineàr integrable systems in $(2+1)$ dimension are generated from a matrix Schrödinger-type inverse problem and solved explicitly to demonstrate a new phenomenon of overturning. Both, the two- and three-dimensional graphical depictions of the solution are presented. Our analysis is an extension of the uncoupled case reported earlier by Bogoyavlenskii. A unique feature of the solution is the occurrence of arbitrary functions of $(y, t)$ in its functional form, which significantly changes the behaviour of the solution.
\end{abstract}

PACS numbers: $02.90 .+\mathrm{p}, 52.35 . \mathrm{Nx}, 52.35 . \mathrm{Sb}$

\section{Introduction}

Solutions in $(2+1)$ dimension were first studied in connection with the Kodomstev-Petviashville equation. Since then several other equations in $(2+1)$ dimension have been analysed in relation to the theory of inverse scattering in multidimension [1]. Many new properties can be seen to be exhibited by such solitons which are not displayed by their counter part in $(1+1)$-dimensional systems. One among such intriguing phenomena is that of overturning which was first discussed by Bogoyavlenskii [2] in the case of an equation in $(2+1)$ dimension which reduces to the Kortewege-de Vries (KdV) equation if we set $x=y$. Here in this paper we have studied a nonlinear coupled system in $(2+1)$ dimension, with the help of a matrix Schrödinger-like linear problem [3]. It is interesting to observe that due to the multidimensional character some arbitrariness remains inherent regarding the dependence of the soliton on the variables $(y, t)$ which, if chosen properly, can lead to a certain interesting phenomenon regarding the structure of the soliton. 


\section{Formulation}

A matrix nonlinear system in $(2+1)$ dimension is written as

$$
U_{t x}=2\left(U_{x}^{2}\right)_{y}-U_{y} U_{x x}+U_{x x} U_{y}-U_{x x x y}-2\left[U_{x}, W\right],
$$

where $W_{x}=\frac{1}{2}\left[U_{x}, U_{y}\right]$ and $[$,$] a commutator. U_{x}$ denotes $\partial U(x, y, t) / \partial x . U$ being a general $2 \times 2$ matrix potential. The Lax pair associated with Eq. (1) can be written as

$$
L \psi=\lambda \psi, \quad L:=-\partial_{x}^{2}+U_{x}(x, y, t), \quad \psi_{t}=A \psi,
$$

where

$$
-\frac{1}{2} A:=\left(\partial_{y} L+L \partial_{y}\right)+V \partial_{x}+\frac{1}{2} v_{x}+W
$$

with $V=U_{y}$ and $W$ as defined above. So the kind of the Lax operators we are considering, belongs to the class discussed by Zakharov [4], where the time part had the general form

$$
A=\sum F_{i}(L) \frac{\partial}{\partial x_{i}}+A,
$$

where $L$ is the space part of the Lax pair containing derivatives with respect to $x$. It may be noted that the compatibility of Eqs. (2) and (3) leads at once to Eq. (1).

Let $\psi(k, t, x, y)$ be the $2 \times 2$ matrix of the Jost function satisfying

$$
L \psi(k, t, x, y)=k^{2} \psi(k, t, x, y) \text {. }
$$

In a matrix form we can rewrite $L$ as

$$
L=-\frac{\partial^{2}}{\partial x^{2}} \delta_{i j}+\left[U_{i j}(t, x, y)\right]_{x},
$$

where $U$ is assumed to have the following asymptotics:

$$
\begin{aligned}
& U(t, x, y) \rightarrow g(t, y), \quad \text { when } x \rightarrow-\infty, \\
& V(t, x, y) \rightarrow h(t, y), \text { when } x \rightarrow+\infty
\end{aligned}
$$

and for the time being $g, h$ are arbitrary $2 \times 2$ matrix functions. We observe that in such case $U_{x} \rightarrow 0$ as $|x| \rightarrow \infty$ and $U_{y}(t, x, y) \rightarrow g_{y}, h_{y}$ for $x \rightarrow \mp \infty$. Furthermore, we assume that $U$ is a continuous and decreasing function of $x$. In the following we summarise some already known features of the inverse scattering procedure for the matrix Schrödinger equation. For real $k$, Jost functions $F, G$ are defined by the asymptotic relations

$$
\begin{aligned}
& \lim _{x \rightarrow+\infty} F(k, t, x, y)=I \exp (\mathrm{i} k x), \\
& \lim _{x \rightarrow-\infty} G(k, t, x, y)=I \exp (-\mathrm{i} k x)
\end{aligned}
$$

(where $I$ denotes the identity matrix) with the usual analyticity arguments remaining valid. Here it is to be remembered that $k=k(y, t)$. Furthermore, the pairs $\{F(k, t, x, y), F(-k, t, x, y)\}$ and $\{G(k, t, x, y), G(-k, t, x, y)\}$ are the fundamental systems of solutions whence the linear dependence of the Jost functions can be expressed as

$$
\begin{aligned}
& G(k, t, x, y)=F(-k, t, x, y) A(k, t, y)+F(k, t, x, y) B(k, t, y) \\
& F(k, t, x, y)=G(-k, t, x, y) C(k, t, y)+G(k, t, x, y) D(k, t, y)
\end{aligned}
$$


The compatibility of Eq. (8) requires

$$
\begin{aligned}
& B(k, t, y) D(k, t, y)+A(-k, t, y) C(k, t, y) \\
& \quad=D(k, t, y) B(k, t, y)+C(-k, t, y) A(k, t, y)=I, \\
& A(k, t, y) D(k, t, y)+B(-k, t, y) C(k, t, y) \\
& \quad=C(k, t, y) B(k, t, y)+D(-k, t, y) A(k, t, y)=0 .
\end{aligned}
$$

It is well known that the coefficients $A, B, C, D$ can be obtained via the Wronskian of $F, F^{*}, G, G^{*}$ from which we observe

$$
\begin{aligned}
& A(k, t, y)=C^{*}(-k, t, y), \\
& B(k, t, y)=-D^{*}(k, t, y) .
\end{aligned}
$$

The bound states of the spectrum which actually correspond to the soliton solution are determined from the condition,

$$
\operatorname{det}\{A(k)\}=0 \text {. }
$$

In the case of a given value $k_{j}$ there will exist a vector $a$ such that

$$
A\left(k_{j}\right) a=0,
$$

whence a solution of (2) can be represented as

$$
\psi\left(x, k_{j}\right)=G\left(x, k_{j}\right) a=F\left(x, k_{j}\right) B\left(k_{j}\right) a .
$$

The asymptotic conditions $(7 \mathrm{a})$ indicate that

$$
\psi\left(x, k_{j}\right) \rightarrow\left\{\begin{array}{cc}
\exp \left(-\mathrm{i} k_{j} x\right) a, & x \rightarrow-\infty \\
\exp \left(\mathrm{i} k_{j} x\right) a, & x \rightarrow+\infty
\end{array}\right.
$$

On the other hand the Hermitian nature of the potential implies that $k_{j}=\mathrm{i} p_{j}$, , where $p_{j}$ is a real positive numbers. So we can infer that the bound states correspond exactly to the points $\operatorname{Im}\{k\}>0, \operatorname{Re}\{k\}=0$, where $\operatorname{det}\{A(k)\}=0$. So that $A^{-1}(k)$ has a pole. From a study of the asymptotic behaviour of the Jost functions in the complex $k$-plane it is not difficult to show that

$$
\begin{aligned}
& A(k) \approx I+O(1 / k), \quad B(k) \approx O(1 / k), \\
& C(k) \approx I+O(1 / k), \quad D(k) \approx O(1 / k),
\end{aligned}
$$

so that the singularities of the matrix $A^{-1}(k)$ in the upper half plane are simple poles and form a finite set. Therefore we have

$$
A^{-1}(k) \approx \frac{N_{j}}{k-k_{j}}+\ldots, \quad N_{j} \neq 0 .
$$




\section{Time evolution}

Since a consistency of Eqs. (2) and (3) expressed as

$$
L_{t}=[L, M]
$$

leads to Eq. (1), we can deduce at once that

$$
\left(L-k^{2}\right)\left(\psi_{t}+M \psi\right)=0 \text {, }
$$

where $k$ is the wave vector. Using the fact that the eigenfunctions $\psi(k, t, x, y)$ depend on two spatial dimensions $x$ and $y$ and the asymptotics

$$
\begin{aligned}
& G(x, k, t, y)=I \exp (\mathrm{i} k x), \quad \text { when } \quad x \rightarrow-\infty, \\
& G(x, k, t, y)=A(k . t, y) \mathrm{e}^{\mathrm{i} k x}+B(k, t, y) \mathrm{e}^{\mathrm{i} k x}, \quad \text { when } x \rightarrow+\infty,
\end{aligned}
$$

where $I$ denotes the identity matrix.

We obtain

$$
\psi_{t}+M \psi=2 \mathrm{i} k g_{y} \psi
$$

which at once leads to

$$
\begin{aligned}
& A_{t}-4 k^{2} A_{y}=2 \mathrm{i} k\left(g_{y}-h_{y}\right) A, \\
& B_{t}-4 k^{2} B_{y}=2 \mathrm{i} k\left(g_{y}+h_{y}\right) B,
\end{aligned}
$$

so that the eigenvalue $f=k^{2}=-p^{2}$ satisfies the equation

$$
\frac{\partial f}{\partial t}-4 f \frac{\partial f}{\partial y}=0 \text {. }
$$

The inverse problem for the matrix Schrödinger problem can now be set up by assuming the Jost function. $F(x, k, t, y)$, as

$$
F(x, k, t, y)=\mathrm{e}^{\mathrm{i} k x} I+\int_{x}^{\infty} K(x, t, y, z) \mathrm{e}^{\mathrm{i} k z} \mathrm{~d} z
$$

then

$$
\frac{\mathrm{d}}{\mathrm{d} x} K(x, t, y, x)=-\frac{1}{2} U_{x}(x, t, y)
$$

with the condition that $K$ satisfies

$$
\left[-\frac{\partial^{2}}{\partial x^{2}}+\frac{\partial^{2}}{\partial z^{2}}+U_{x}(x, t, y)\right] K(x, t, y)=0 .
$$

Operating with $A^{-1}$ from the right on Eq. (8), we get

$$
G(x, k, t, y) A^{-1}(k, t, y)=F(x,-k, t, y)+F(x, k, t, y) R(k, t, y),
$$

where

$$
R(k, t, y)=B(k, t, y) A^{-1}(k, t, y) .
$$

Taking the Fourier transform of Eq. (23a) and using Eqs. (21) we get

$$
K(x, t, y, z)+H(t, y, x+z)+\int_{x}^{\infty} \mathrm{d} s K(x, s) H(z+s)=0 ;
$$

where

$$
H(t, y, x)=\frac{1}{2 \pi} \int_{-\infty}^{\infty} \mathrm{d} k R(k, t, y) \mathrm{e}^{\mathrm{i} k x}-\mathrm{i} \sum_{j=1}^{s} R_{j} \mathrm{e}^{\mathrm{i} k k_{j} x} .
$$

This way Eqs. (24) and (25) form the basic equations of the inverse problem for the matrix Schrödinger equation. 


\section{Construction of one soliton solution}

Keeping only the discrete part in Eq. (25) corresponding to the single pole in $R$ we can at once solve Eq. (24) and obtain the following integral equations in the component form

$$
\begin{aligned}
& K_{11}+H_{11}+\int_{x}^{\infty} \mathrm{d} s\left(K_{11} H_{11}+K_{12} H_{21}\right)=0, \\
& K_{12}+H_{12}+\int_{x}^{\infty} \mathrm{d} s\left(K_{11} H_{12}+K_{12} H_{22}\right)=0, \\
& K_{21}+H_{21}+\int_{x}^{\infty} \mathrm{d} s\left(K_{21} H_{11}+K_{22} H_{21}\right)=0, \\
& K_{22}+H_{22}+\int_{x}^{\infty} \mathrm{d} s\left(K_{21} H_{12}+K_{22} H_{22}\right)=0,
\end{aligned}
$$

which yield

$$
L_{11}(x)=\frac{-\alpha \mathrm{e}^{\mathrm{i} k_{j} x}+\left(\frac{\alpha \delta-\gamma \beta}{2 \mathrm{i} k_{j}}\right) \mathrm{e}^{3 \mathrm{i} k_{j} x}}{1-\frac{\alpha+\delta}{2 i k_{j}} \mathrm{e}^{2 \mathrm{i} k_{j} x}+\left(\frac{\alpha \delta-\gamma \beta}{4 k_{j}^{2}}\right) \mathrm{e}^{4 \mathrm{i} k_{j} x}}
$$

with similar expressions for $L_{12}, L_{21}$ and $L_{22}$. The parameters $(\alpha, \beta, \gamma, \delta)$ are expressed by $A_{i j}, B_{i j}$ in the following way:

$$
\begin{gathered}
\alpha=-\frac{B_{11} A_{22}-B_{12} A_{21}}{A_{11} A_{22}-A_{12} A_{21}}, \quad \beta=\frac{B_{12} A_{11}-B_{11} A_{12}}{A_{11} A_{22}-A_{12} A_{21}}, \\
\gamma=\frac{B_{22} A_{21}-B_{21} A_{22}}{A_{11} A_{22}-A_{12} A_{21}}, \quad \delta=\frac{B_{22} A_{11}-B_{21} A_{12}}{A_{11} A_{22}-A_{12} A_{21}} .
\end{gathered}
$$

Now, if we refer back to Eq. (20) we observe that the time evolution of $A_{i j}$ and $B_{i j}$ can be obtained as

$$
\begin{aligned}
& A_{i j}= \begin{cases}\exp \left(-\int c_{0}^{1 / 2}\left(g_{1 y}-h_{1 y}\right) \mathrm{d} y+\chi_{i j}\left(c_{0}\right)\right) & \text { when } i=1, j=1,2, \\
\exp \left(-\int c_{0}^{1 / 2}\left(g_{2 y}-h_{2 y}\right) \mathrm{d} y+\chi_{i j}\left(c_{0}\right)\right) & \text { when } i=2, j=1,2,\end{cases} \\
& B_{i j}= \begin{cases}\exp \left(-\int c_{0}^{1 / 2}\left(g_{1 y}+h_{1 y}\right) \mathrm{d} y+\tau_{i j}\left(c_{0}\right)\right) & \text { when } i=1, j=1,2, \\
\exp \left(-\int c_{0}^{1 / 2}\left(g_{2 y}+h_{2 y}\right) \mathrm{d} y+\tau_{i j}\left(c_{0}\right)\right) & \text { when } i=2, j=1,2,\end{cases}
\end{aligned}
$$

$X_{i j}, C_{i j}$ are arbitrary functions and $C_{0}=(t+1) /(y+1)$ with the conditions

$$
g_{1 y}=G(y)(t+1)^{1 / 2}, \quad h_{1 y}=H(y)(t+1)^{1 / 2} .
$$

Equations (26), (27), (28) and (30) determine the form of the soliton in (2+1) space-time. 


\section{Form of the soliton and overturning}

To understand the behaviour of this soliton solution we analyse its behaviour graphically. In Fig. 1 we have depicted a two-dimensional section of the solution given by Eq. (27) for the choice of the functions and the parameters as specified. It is easy to observe that the soliton suddenly turns around and becomes peaked in the reverse direction. In Figs. $2 \mathrm{a}$ and $\mathrm{b}$ we show a corresponding three-dimensional plot at $t=0$, which also exhibits the same phenomenon. To study the propagation of such a wave-form we next consider its form at $t=0.75$ and display its subsequent form in Figs. $3 \mathrm{a}$ and $\mathrm{b}$. The corresponding forms for the negative values, at $t=$ -0.75 are shown in Figs. $4 \mathrm{a}$ and $\mathrm{b}$. Note that for these diagrams we have chosen $g_{1 y}=-h_{1 y}=y(t+1)^{-1 / 2}$. On the other hand a different choice of these functions is also possible and we have considered the case $g_{1 y}=-h_{1 y}=[(y+1) /(t+1)]^{1 / 2}$, whence one observes that the distinct overturning behaviour disappears and the two- and three-dimensional forms are depicted in Figs. $5 \mathrm{a}$ and $\mathrm{b}$. This shows that the functional behaviour of the soliton depends on $y$ and $t$ can be adjusted to change its characteristics.
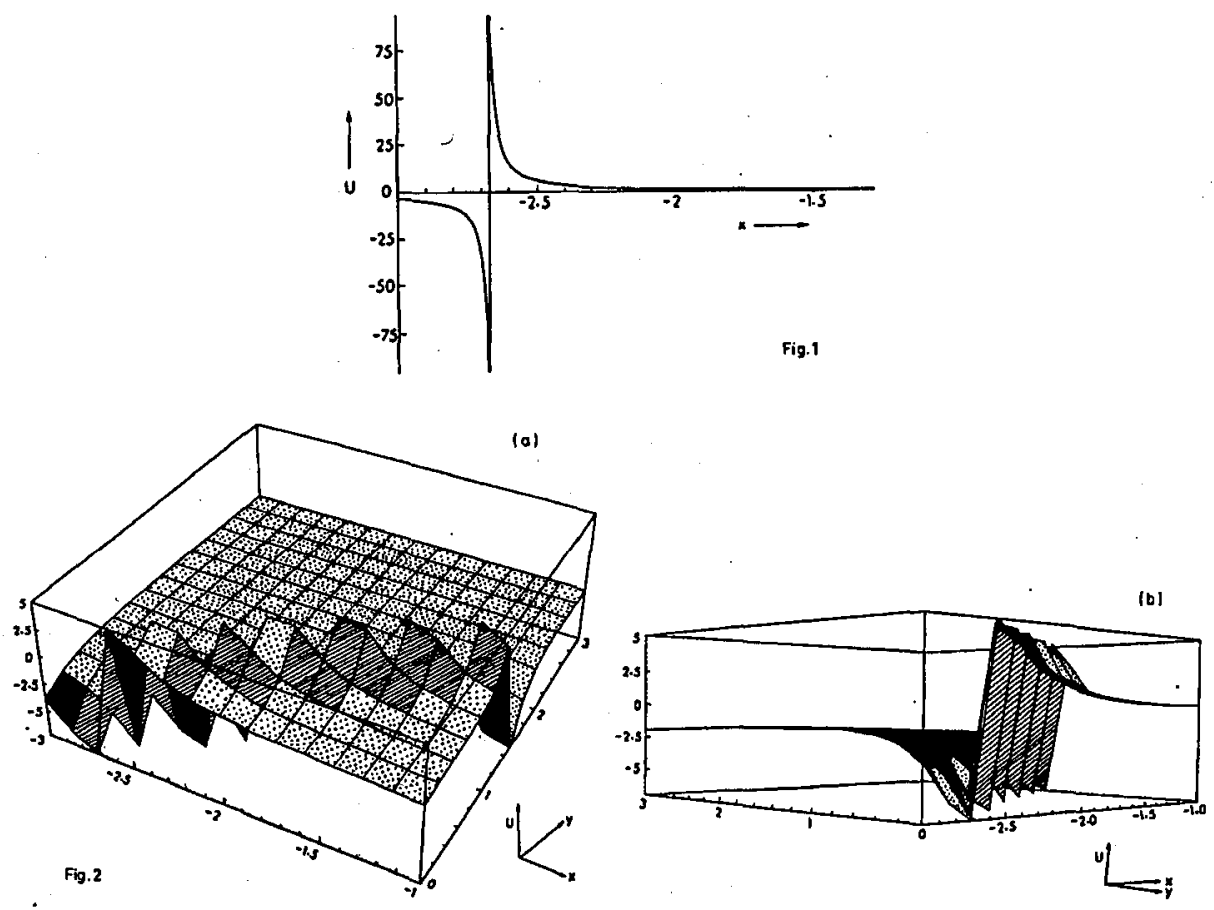

Fig. 1. Plot of $U$ as a function of $x$ at $t=0$ and $y=0$, when $g_{1 y}=-h_{1 y}=y(t+1)^{-1 / 2}$. Fig. 2. Plot of $U$ as a function of $x$ and $y$ at $t=0$, for $x=-1$ to -3 and $y=0$ to 3 (a) and from the left hand corner for $x=-1$ to -3 and $y=0$ to 3 (b). 

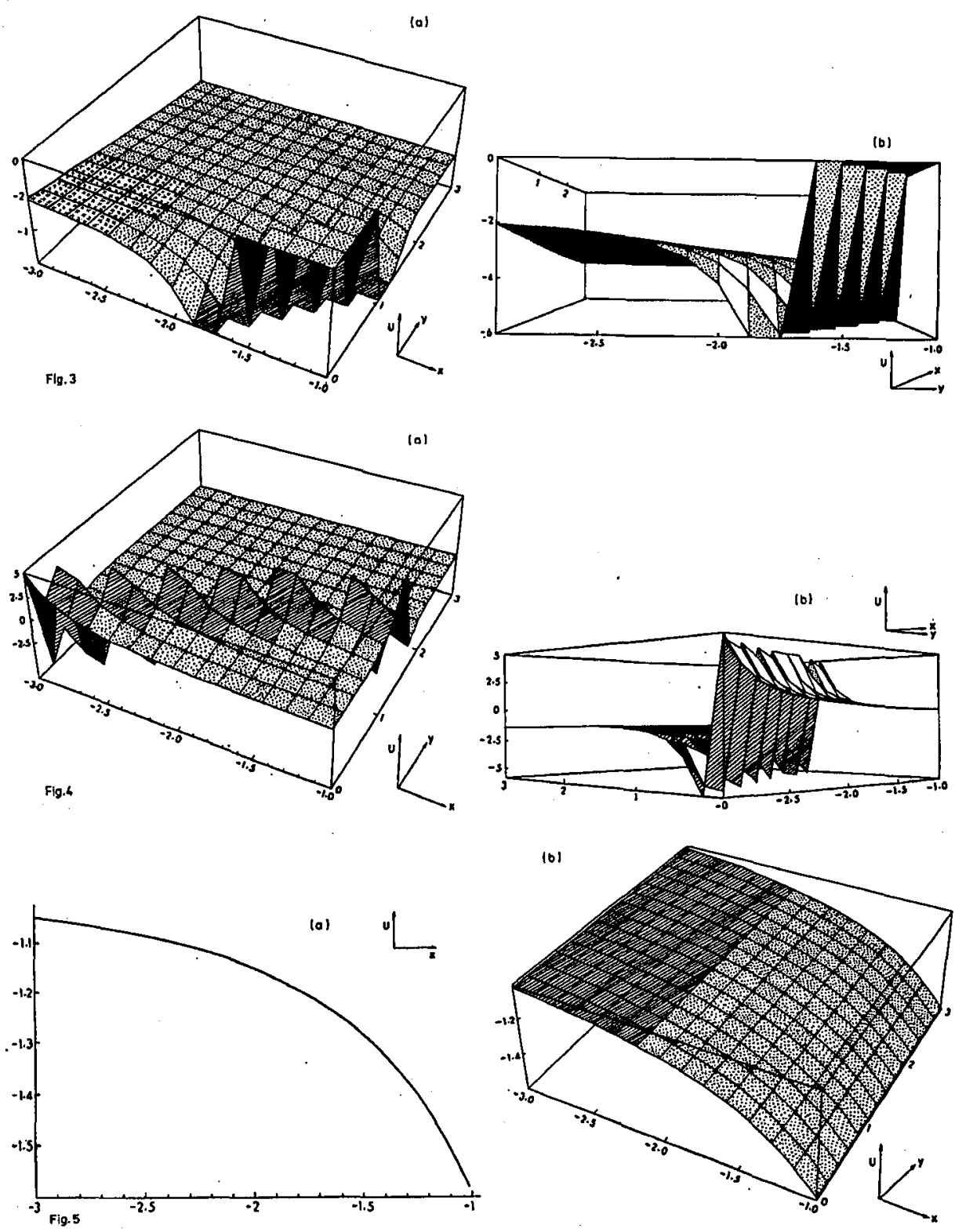

Fig. 3. Plot of $U$ as a function of $x$ and $y$ at $t=0.75$, for $x=-1$ to -3 and $y=0$ to 3 , when $g_{1 y}=-h_{1 y}=y(t+1)^{-1 / 2}$ (a) and from directly in front for $x=-1$ to -3 and $y=0$ to 3 (b).

Fig. 4 Plot of $U$ as a function of $x$ and $y$ at $t=-0.75$, for $x=-1$ to -3 and $y=0$ to 3 , when $g_{1 y}=-h_{1 y}=y(t+1)^{-1 / 2}$ (a) and from the left hand corner for $x=-1$ to -3 and $y=0$ to 3 (b).

Fig. 5 Plot of $U$ as a function of $x$ at $t=0, y=0$, when $g_{1 y}=h_{1 y}=[(y+1) /(t+1)]^{1 / 2}$ (a) and as a function of $x$ and $y$ at $t=0$ for $x=-1$ to -3 and $y=0$ to 3 (b). 


\section{Discussions}

In our analysis above we have shown a new type of behaviour for the case of a solitary wave in $(2+1)$ space-time. It is also interesting to note that such behaviour can be changed by changing the arbitrariness of the dependence on $(y, t)$ coordinates.

One of the authors (C.G.) is grateful to C.S.I.R. for a fellowship.

\section{References}

[1] M.J. Ablowitź, P.A. Clarkson, Solitons, Nonlinear Evolution Equations and Inverse Scattering, London Math. Society, Vol. 149, Cambridge Univ. Press, London 1991.

[2] O.I. Bogoyavlenskii, Math. USSR Izvestiya 34, 245 (1990).

[3] F. Calogero, Spectral Transform and Solitons I, North-Holland, Amsterdam 1982.

[4] V.E. Zakharov, in: Solitons, Topics in Current Physics, Eds. R.K. Bullough, P.J. Caudrey, Springer-Verlag, Berlin 1980. 